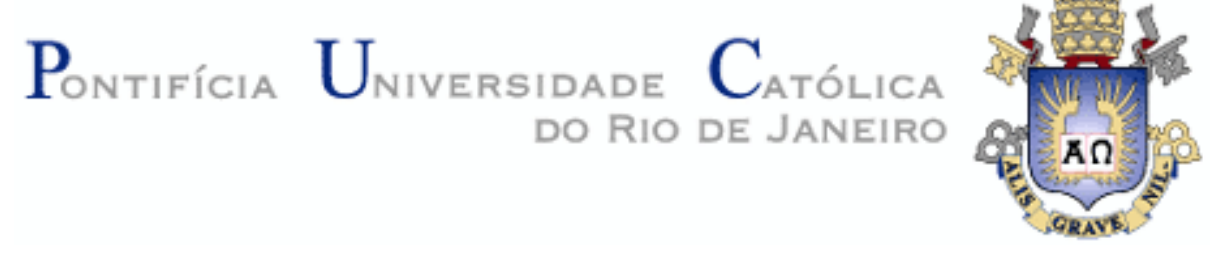

Juliana Kuhlmann Abrantes

\title{
Estudo do Escoamento e Transferência de Calor em um Jato Espiralado Incidente
}

Dissertação de Mestrado

Dissertação apresentada como requisito parcial para obtenção do título de Mestre pelo Programa de PósGraduação em Engenharia Mecânica da PUC-Rio.

Orientador: Prof. Luis Fernando Alzuguir Azevedo

Rio de Janeiro

Abril de 2005 


\section{Estudo do Escoamento e Transferência de Calor em um Jato Espiralado Incidente}

Dissertação apresentada como requisito parcial para obtenção do título de Mestre pelo Programa de PósGraduação em Engenharia Mecânica da PUC-Rio. Aprovada pela Comissão Examinadora abaixo assinada.

Luis Fernando Alzuguir Azevedo Orientador

Pontifícia Universidade Católica do Rio de Janeiro

Angela Ourivio Nieckele

Pontifícia Universidade Católica do Rio de Janeiro

Marcos Sebastião de Paula Gomes Pontifícia Universidade Católica do Rio de Janeiro

Aristeu da Silveira Neto Universidade Federal de Uberlândia

José Eugenio Leal

Coordenador Setorial do Centro Técnico Científico - PUC-Rio

Rio de Janeiro, 11 de abril de 2005 
Todos os direitos reservados. É proibida a reprodução total ou parcial do trabalho sem autorização da universidade, da autora e do orientador.

\section{Juliana Kuhlmann Abrantes}

Graduou-se em Engenharia Mecânica e Engenharia de Produção Mecânica na Pontifícia Universidade Católica do Rio de Janeiro (Rio de Janeiro, Brasil) em 2002.

Ficha Catalográfica

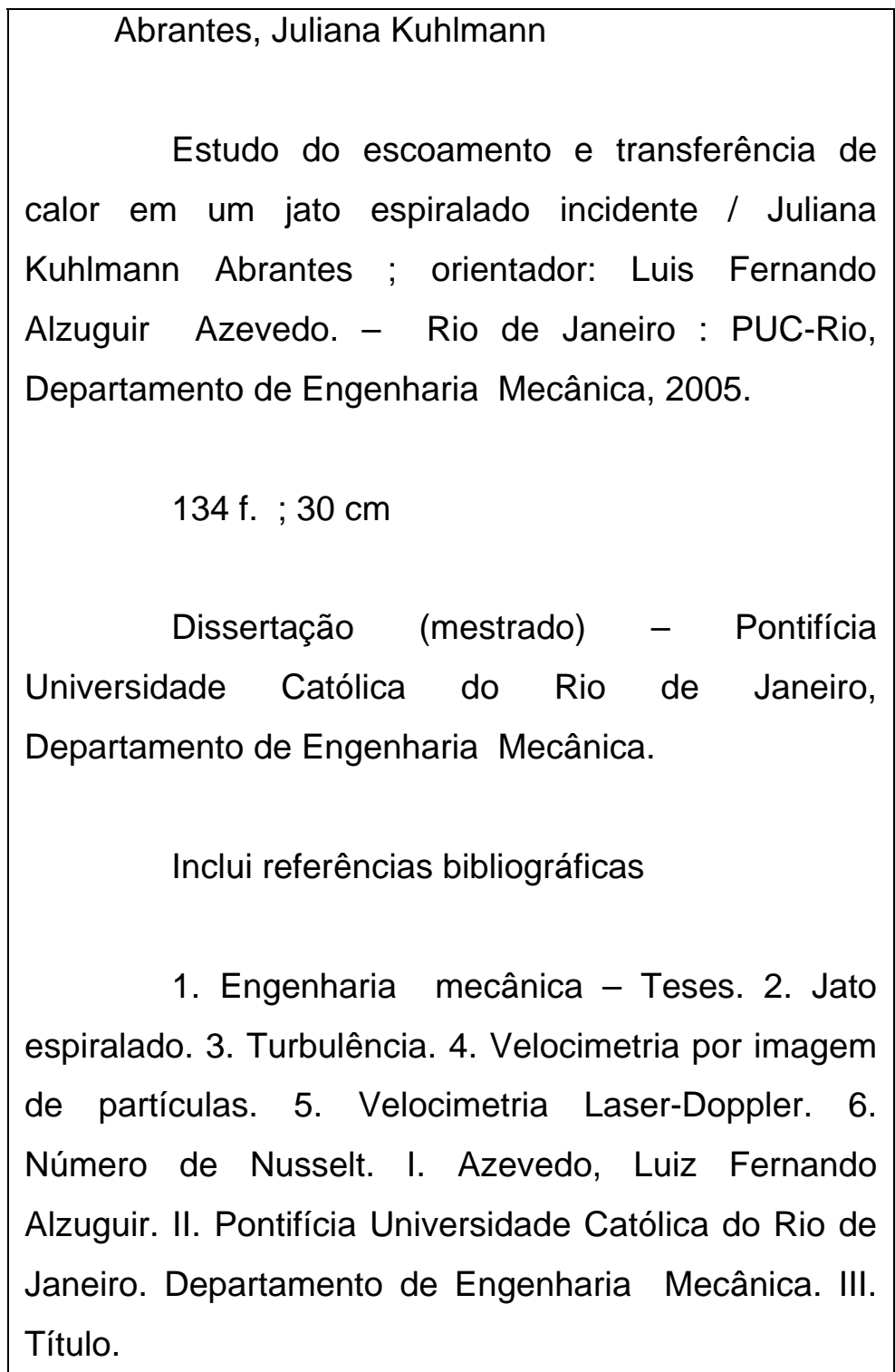

CDD: 621 


\section{Agradecimentos}

Ao meu orientador, Professor Luis Fernando A. Azevedo, pela dedicação, paciência e incentivo constante.

Aos professores e funcionários do Departamento de Engenharia Mecânica da PUC-Rio que contribuíram para a realização deste trabalho.

Agradeço também aos Professores membros da banca, pela participação, comentários e sugestões feitas ao trabalho apresentado.

Aos meus pais e a toda a minha família pelo apoio e carinho sempre manifestados.

A todos os amigos do Laboratório de Termociências da PUC-Rio, que contribuíram de diferentes maneiras para o sucesso deste trabalho.

Finalmente, agradeço ao Cnpq e à Faperj pelos auxílios concedidos. 


\section{Resumo}

Abrantes, Juliana Kuhlmann. Estudo do Escoamento e Transferência de Calor em um Jato Espiralado Incidente. Rio de Janeiro, 2005. 134p. Dissertação de Mestrado - Departamento de Engenharia Mecânica, Pontifícia Universidade Católica do Rio de Janeiro.

O presente trabalho é um estudo experimental das características de um escoamento de ar em forma de jato espiralado, incidindo ortogonalmente sobre uma placa. Os objetivos do estudo são: avaliar a influência da presença de uma componente circunferencial de velocidade na distribuição dos coeficientes locais de troca de calor, obter campos de velocidade instantâneos no plano axissimétrico assim como informações sobre as características da turbulência no escoamento. Durante os experimentos se investigou a influência da distância jato/placa e da intensidade do escoamento espiralado (número de Swirl). Como etapa preliminar, foi conduzido um experimento de jato livre, para validação das técnicas de medição de velocidade utilizadas. Os resultados foram comparados com os da literartura e uma boa concordância foi obtida. A distribuição espacial dos coeficientes de troca de calor foi avaliada impondo-se um fluxo de calor constante na placa e medindo a distribuição radial de temperatura através de diversos termopares. Coeficientes locais puderam então ser estimados. Os campos de velocidades radial e axial instantâneos foram adquiridos experimentalmente através da utilização da técnica de "Particle Image Velocimetry" (PIV) e perfis de velocidade tangencial (média e flutuações) foram obtidos a partir da técnica "Laser Doppler Velocimetry" (LDV). Os resultados mostraram que os padrões de escoamento mudam significativamente quando a componente circunferencial de velocidade é introduzida. Para o valor mais alto do Número de Swirl foram verificadas fortes reversões do escoamento na região de estagnação.

\section{Palavras-chave}

Jato Espiralado; Turbulência; Velocimetria por Imagem de Partículas; Velocimetria Laser-Doppler; Número de Nusselt 


\section{Abstract}

Abrantes, Juliana Kuhlmann. Study of Flow and Heat Transfer Characteristics in a Swirling Impinging Jet. Rio de Janeiro, 2005. 134p. Dissertação de Mestrado - Departamento de Engenharia Mecânica, Pontifícia Universidade Católica do Rio de Janeiro.

The present work is an experimental study of the characteristics of a swirling impinging air jet. The goals of the study are: to evaluate the influence of the presence of a circumferential velocity component in the distribution of the local heat transfer coefficients, to obtain instantaneous velocity fields in the axisymmetric plane, as well as information about the turbulence characteristics in the flow. During the experiments, the influence of the impingement distance and swirl intensity were investigated. As a preliminary validation of the velocity measurement tecniques, an experimental investigation of an axisymmetric free jet was conducted. The results were compared with literature showing good agreement. The spatial distribution of heat transfer coefficients was evaluated by imposing a constant heat flux condition to the plate and measuring temperature of several points along the radial distance of the plate with thermocouples. Local coefficients could then be estimated. Instantaneous axial and radial velocity fields were obtained with "Particle Image Velocimetry" (PIV) and tangential velocity profiles (mean and fluctuations) obtained by using "Laser Doppler Velocimetry" (LDV). The results showed that the flow patterns change significantly when the tangential component is added. For the highest value of Swirl number, strong recirculation zones were observed in the stagnation region.

\section{Keywords}

Swirling Jet; Turbulence; Particle Image Velocimetry; Laser-Doppler Velocimetry; Nusselt Number. 


\section{Sumário}

$\begin{array}{ll}1 \text { Introdução } & 17\end{array}$

$\begin{array}{ll}2 \text { Revisão Bibliográfica } & 20\end{array}$

3 Descrição dos Experimentos 25

3.1. Câmara de Testes 28

3.2. Seções Geradora e Reguladora de Escoamento Espiralado 28

3.3. Placa e Suporte 32

$\begin{array}{ll}\text { 3.4. Outros Componentes } & 37\end{array}$

4 Técnicas de Medição do Escoamento 44

4.1. Velocimetria por Imagem de Partículas - PIV 44

4.2. Velocimetria Laser-Doppler - LDV 57

5 Caracterização dos Parâmetros do Experimento 61

5.1. Determinação do Número de Swirl 61

5.2. Determinação do Número de Reynolds 62

6 Procedimento Experimental 63

6.1. Medição com PIV 63

6.2. Medição com LDV 65

6.3. Medidas de Transferência de Calor 66

7 Tratamento dos Dados $\quad 68$

7.1. Coeficientes de Troca de Calor 68

$\begin{array}{ll}\text { 7.2. Campo de Velocidades } & 69\end{array}$

8 Resultados para Jato Livre: Validação da Técnica LDV 73 
9 Resultados e Discussão $\quad 87$

9.1. Visão Geral da Estrutura do Escoamento 88

9.2. Efeitos da Estrutura do Escoamento na Transferência de Calor 105

9.3. Apresentação de Perfis de Velocidade e Quantidades Turbulentas 108

10 Conclusões

Referências Bibliográficas 


\section{Lista de Figuras}

1.1 Representação esquemática de um jato incidindo $\begin{array}{ll}\text { sobre uma superfície plana } & 18\end{array}$

2.1 Visualização da superfície atingida pelo jato espiralado [1] 22

2.2 Visualização da região de estagnação do jato espiralado [1] 23

2.3 Padrão de escoamento esperado para a região de estagnação do jato espiralado $\quad 23$

3.1 Visão geral da seção de testes 26

3.2 Seção de testes em experimento de medição de velocidade com LDV $\quad 27$

3.3 Seção de testes preparada para experimento de transferência de calor $\quad 27$

3.4 Detalhe da seção geradora de escoamento espiralado 29

3.5 Montagem da seção geradora de escoamento espiralado 29

3.6 Montagem da seção geradora com cilindro externo 30

3.7 Montagem completa utilizada para a geração do jato espiralado 31

3.8 Acoplamento dos tubos de cobre 31

3.9 Luva centralizadora para os tubos de cobre 32

3.10 Detalhe da placa de vidro durante medição com a técnica "Laser Doppler Velocimetry" (LDV)

3.11 Suporte da placa de vidro utilizada nos experimentos de medição do escoamento

3.12 Vista esquemática da placa aquecedora utilizada nos experimentos de transferência de calor $\quad 35$

3.13 Vista superior da placa aquecedora 36

3.14 Vista lateral da placa aquecedora 36

3.15 Furação na placa de celeron para posicionamento dos termopares

3.16 Suporte da placa utilizada nos experimentos de transferência de calor 
3.17 Processo de centralização para os experimentos de transferência de calor 39

3.18 Gerador de Partículas Traçadoras [17] 41

3.19 Detalhe do bocal "Laskin" 41

3.20 Distribuição do tamanho das partículas geradas 42

4.1 Diagrama de sincronismo utilizado na aquisição de imagens com PIV $\quad 49$

4.2 Regiões base e de busca para correlação cruzada 50

4.3 Posição do pico de correlação máximo 51

4.4 Mapa típico do coeficiente de correlação, R, para correlação cruzada

4.5 Função Densidade de Probabilidade do deslocamento de partículas - Efeito de "peak-locking" [4]

4.6 Movimento das franjas em um sistema LDV com "frequency shift"

4.7 Deslocamento de freqüência para a medição de reversões no escoamento

6.1 Típica imagem das partículas em um experimento com PIV para um jato incidente

8.1 Variação da velocidade média axial na linha de centro de um jato livre, $\mathrm{Re}=21000$

8.2 Perfil de velocidade média na saída do tubo, $\mathrm{Re}=21000$

8.3 Perfil médio de velocidade axial para jato livre, $\mathrm{Re}=21000 \quad 80$

8.4 Parcela axial de Energia Cinética Turbulenta no jato livre, $\mathrm{Re}=21000$

8.5 Parcela radial de Energia Cinética Turbulenta no jato livre, $\mathrm{Re}=21000$

8.6 Parcela azimutal de Energia Cinética Turbulenta no jato livre, $\mathrm{Re}=21000$

8.7 Tensão cisalhante turbulenta no jato livre, $\mathrm{Re}=21000$

8.8 Tensão cisalhante turbulenta: comparação entre dados experimentais diretos e perfil calculado pela equação (8-13) 84

9.1 Campo Médio de Velocidade, $\mathrm{Re}=21000, \mathrm{H} / \mathrm{d}=2, \mathrm{~S}=0$ 
9.2 Campos Instantâneos de Velocidade, freqüência de amostragem $=15 \mathrm{~Hz}, \mathrm{Re}=21000, \mathrm{H} / \mathrm{d}=2, \mathrm{~S}=0$

9.3 Campo Médio de Velocidade, $R e=21000, H / d=2, S=0.3$

9.4 Campos Instantâneos de Velocidade, freqüência de amostragem $=15 \mathrm{~Hz}, \mathrm{Re}=21000, \mathrm{H} / \mathrm{d}=2, \mathrm{~S}=0.3$

9.5 Campo Médio de Velocidade, $R e=21000, H / d=2, S=0.5$

9.6 Campos Instantâneos de Velocidade, freqüência de amostragem $=15 \mathrm{~Hz}, \mathrm{Re}=21000, \mathrm{H} / \mathrm{d}=2, \mathrm{~S}=0.5$

9.7 Contornos de Magnitude de Velocidade Média [m/s] com Linhas de Corrente, $\mathrm{Re}=21000, \mathrm{H} / \mathrm{d}=2, \mathrm{~S}=0$

9.8 Contornos de Magnitude de Velocidade Média $[\mathrm{m} / \mathrm{s}]$ com Linhas de Corrente, $\mathrm{Re}=21000, \mathrm{H} / \mathrm{d}=2, \mathrm{~S}=0.3$

9.9 Contornos de Magnitude de Velocidade Média $[\mathrm{m} / \mathrm{s}]$ com Linhas de Corrente, $\mathrm{Re}=21000, \mathrm{H} / \mathrm{d}=2, \mathrm{~S}=0.5$

9.10 Contornos de Magnitude de Velocidade Média, $\sqrt{\bar{U}^{2}+\bar{V}^{2}},[\mathrm{~m} / \mathrm{s}]$, $\mathrm{Re}=21000, \mathrm{H} / \mathrm{d}=2, \mathrm{~S}=0$

9.11 Contornos de Velocidade Axial Turbulenta r.m.s. - $\boldsymbol{u}^{\prime}$ [m/s] $\mathrm{Re}=21000, \mathrm{H} / \mathrm{d}=2, \mathrm{~S}=0$

9.12 Contornos de Velocidade Radial Turbulenta r.m.s. - $\boldsymbol{v}$ ' [m/s] $\mathrm{Re}=21000, \mathrm{H} / \mathrm{d}=2, \mathrm{~S}=0$

9.13 Contornos de Magnitude de Velocidade Média, $\sqrt{\bar{U}^{2}+\bar{V}^{2}},[\mathrm{~m} / \mathrm{s}], \mathrm{Re}=21000, \mathrm{H} / \mathrm{d}=2, \mathrm{~S}=0.3$

9.14 Contornos de Velocidade Axial Turbulenta r.m.s., $\boldsymbol{u}^{\prime}[\mathrm{m} / \mathrm{s}]$ $\mathrm{Re}=21000, \mathrm{H} / \mathrm{d}=2, \mathrm{~S}=0.3$

9.15 Contornos de Velocidade Radial Turbulenta r.m.s., $\boldsymbol{v}^{\prime}$ [m/s] $\mathrm{Re}=21000, \mathrm{H} / \mathrm{d}=2, \mathrm{~S}=0.3$

9.16 Contornos de Magnitude de Velocidade Média, $\sqrt{\bar{U}^{2}+\bar{V}^{2}},[\mathrm{~m} / \mathrm{s}], \mathrm{Re}=21000, \mathrm{H} / \mathrm{d}=2, \mathrm{~S}=0.5$

9.17 Contornos de Velocidade Axial Turbulenta, $\boldsymbol{u}^{\prime}[\mathrm{m} / \mathrm{s}]$ $\mathrm{Re}=21000, \mathrm{H} / \mathrm{d}=2, \mathrm{~S}=0.5$

9.18 Contornos de Velocidade Radial Turbulenta, $\boldsymbol{v}^{\prime}[\mathrm{m} / \mathrm{s}]$ $\mathrm{Re}=21000, \mathrm{H} / \mathrm{d}=2, \mathrm{~S}=0.5$ 102

9.19 Contornos de Magnitude de Velocidade Média, 
$\sqrt{\bar{U}^{2}+\bar{V}^{2}}[\mathrm{~m} / \mathrm{s}], \mathrm{Re}=21000, \mathrm{H} / \mathrm{d}=6, \mathrm{~S}=0$

9.20 Contornos de Velocidade Axial Turbulenta r.m.s., u' [m/s] $\mathrm{Re}=21000, \mathrm{H} / \mathrm{d}=6, \mathrm{~S}=0$

9.21 Contornos de Velocidade Radial Turbulenta r.m.s., $\boldsymbol{v}^{\prime}$ [m/s] $\mathrm{Re}=21000, \mathrm{H} / \mathrm{d}=6, \mathrm{~S}=0$ 103

9.22 Contornos de Magnitude de Velocidade Média, $\sqrt{\bar{U}^{2}+\bar{V}^{2}},[\mathrm{~m} / \mathrm{s}]$ $\mathrm{Re}=21000, \mathrm{H} / \mathrm{d}=6, \mathrm{~S}=0.3$ 103

9.23 Contornos de Velocidade Axial Turbulenta r.m.s., u' [m/s] $\mathrm{Re}=21000, \mathrm{H} / \mathrm{d}=6, \mathrm{~S}=0.3$ 104

9.24 Contornos de Velocidade Radial Turbulenta r.m.s., $\boldsymbol{v}^{\prime}$ [m/s] $\mathrm{Re}=21000, \mathrm{H} / \mathrm{d}=6, \mathrm{~S}=0.3$

9.25 Contornos de Magnitude de Velocidade Média, $\sqrt{\bar{U}^{2}+\bar{V}^{2}},[\mathrm{~m} / \mathrm{s}]$, $\mathrm{Re}=21000, \mathrm{H} / \mathrm{d}=6, \mathrm{~S}=0.5$ 104

9.26 Contornos de Velocidade Axial Turbulenta r.m.s., $\boldsymbol{u}^{\prime}[\mathrm{m} / \mathrm{s}]$ $\mathrm{Re}=21000, \mathrm{H} / \mathrm{d}=6, \mathrm{~S}=0.5$ 105

9.27 Contornos de Velocidade Radial Turbulenta r.m.s., $\boldsymbol{v}^{\prime}$ [m/s] $\mathrm{Re}=21000, \mathrm{H} / \mathrm{d}=6, \mathrm{~S}=0.5$

9.28 Distribuição Radial do Número de Nusselt local $\mathbf{N u}$ $\mathrm{Re}=21000, \mathrm{H} / \mathrm{d}=2$ 106

9.29 Distribuição Radial do Número de Nusselt local $\mathrm{Nu}$ $\mathrm{Re}=21000, \mathrm{H} / \mathrm{d}=6$

9.30 Esquema ilustrativo do sistema de coordenadas utilizado para o jato incidente

9.31 Perfis de Velocidade Radial Média $\mathrm{Re}=21000, \mathrm{H} / \mathrm{d}=2, \mathrm{~S}=0$

9.32 Perfis de Velocidade Radial Turbulenta r.m.s.

$$
\mathrm{Re}=21000, \mathrm{H} / \mathrm{d}=2, \mathrm{~S}=0
$$

9.33 Perfis de Velocidade Axial Turbulenta r.m.s.

$$
\mathrm{Re}=21000, \mathrm{H} / \mathrm{d}=2, \mathrm{~S}=0
$$

9.34 Perfis de Tensão Cisalhante Turbulenta

$$
\mathrm{Re}=21000, \mathrm{H} / \mathrm{d}=2, \mathrm{~S}=0
$$

9.35 Variação radial das velocidades turbulentas r.m.s. perto da parede, $(y=0.01 d), R e=21000, H / d=2, S=0$ 
9.36 Perfis de Velocidade Radial Média

$$
\mathrm{Re}=21000, \mathrm{H} / \mathrm{d}=2, \mathrm{~S}=0.3
$$

9.37 Perfis de Velocidade Radial Turbulenta r.m.s.

$$
\mathrm{Re}=21000, \mathrm{H} / \mathrm{d}=2, \mathrm{~S}=0.3
$$

9.38 Perfis de Velocidade Axial Turbulenta r.m.s.

$$
\mathrm{Re}=21000, \mathrm{H} / \mathrm{d}=2, \mathrm{~S}=0.3
$$

9.39 Variação radial das velocidades turbulentas r.m.s.

perto da parede, $(y=0.01 d), R e=21000, H / d=2, S=0.3$

9.40 Perfis de Velocidade Radial Média

$$
\mathrm{Re}=21000, \mathrm{H} / \mathrm{d}=2, \mathrm{~S}=0.5
$$

9.41 Perfis de Velocidade Tangencial Média

$$
\mathrm{Re}=21000, \mathrm{H} / \mathrm{d}=2, \mathrm{~S}=0.5
$$

9.42 Perfis de Velocidade Radial Turbulenta r.m.s

$$
\mathrm{Re}=21000, \mathrm{H} / \mathrm{d}=2, \mathrm{~S}=0.5
$$

9.43 Perfis de Velocidade Axial Turbulenta r.m.s.

$\mathrm{Re}=21000, \mathrm{H} / \mathrm{d}=2, \mathrm{~S}=0.5$

9.44 Perfis de Velocidade Tangencial Turbulenta r.m.s.

$\mathrm{Re}=21000, \mathrm{H} / \mathrm{d}=2, \mathrm{~S}=0.5$

9.45 Variação Radial das velocidades turbulentas r.m.s. perto da parede $(\mathrm{y}=0.02 \mathrm{~d}), \mathrm{Re}=21000, \mathrm{H} / \mathrm{d}=2, \mathrm{~S}=0.5$

9.46 Variação Radial de Energia Cinética Turbulenta k perto da parede $(y=0.02 d), R e=21000, H / d=2, S=0.5$

9.47 Perfis de Velocidade Radial Média

$\mathrm{Re}=21000, \mathrm{H} / \mathrm{d}=6, \mathrm{~S}=0$

9.48 Perfis de Velocidade Radial Turbulenta r.m.s.

$\mathrm{Re}=21000, \mathrm{H} / \mathrm{d}=6, \mathrm{~S}=0$

9.49 Perfis de Velocidade Axial Turbulenta r.m.s.

$\mathrm{Re}=21000, \mathrm{H} / \mathrm{d}=6, \mathrm{~S}=0$

9.50 Perfis de Tensão Cisalhante Turbulenta

$\mathrm{Re}=21000, \mathrm{H} / \mathrm{d}=6, \mathrm{~S}=0$

9.51 Variação Radial das Velocidades Turbulentas r.m.s. perto da parede, $(y=0.01 d), R e=21000, H / d=6, S=0$

9.52 Perfis de Velocidade Radial Média 
$\mathrm{Re}=21000, \mathrm{H} / \mathrm{d}=6, \mathrm{~S}=0.3$

9.53 Perfis de Velocidade Radial Turbulenta r.m.s.

$\mathrm{Re}=21000, \mathrm{H} / \mathrm{d}=6, \mathrm{~S}=0.3$

9.54 Perfis de Velocidade Axial Turbulenta r.m.s.

$\mathrm{Re}=21000, \mathrm{H} / \mathrm{d}=6, \mathrm{~S}=0.3$

124

9.55 Variação Radial das Velocidades Turbulentas r.m.s.

perto da parede $(\mathrm{y}=0.02 \mathrm{~d}), \mathrm{Re}=21000, \mathrm{H} / \mathrm{d}=6, \mathrm{~S}=0.3$

9.56 Perfis de Velocidade Radial Média

$\mathrm{Re}=21000, \mathrm{H} / \mathrm{d}=6, \mathrm{~S}=0.5$

9.57 Perfis de Velocidade Radial Turbulenta r.m.s.

$\mathrm{Re}=21000, \mathrm{H} / \mathrm{d}=6, \mathrm{~S}=0.5$

9.58 Perfis de Velocidade Axial Turbulenta r.m.s.

$\mathrm{Re}=21000, \mathrm{H} / \mathrm{d}=6, \mathrm{~S}=0.5$

9.59 Variação Radial das Velocidades Turbulentas r.m.s. perto da parede $(y=0.02 d), R e=21000, H / d=6, S=0.5$ 


\section{Lista de Símbolos}

$A_{S} \quad$ Área superficial da placa $\left[\mathrm{m}^{2}\right]$

$b \quad$ Largura da folha de aço inoxidável [mm]

d Diâmetro do tubo do jato [mm]

$d_{\text {diff }}$ Diâmetro limitado por difração $[\mu \mathrm{m}]$

$d_{f} \quad$ Espaçamento entre as franjas óticas do volume de medição

$d_{m} \quad$ Diâmetro médio do volume de medição com LDV [ $\left.\mu \mathrm{m}\right]$

$d_{p} \quad$ Diâmetro das partículas traçadoras $[\mu \mathrm{m}]$

$d_{\tau} \quad$ Diâmetro das partículas traçadoras na imagem $[\mu \mathrm{m}]$

$f_{s h} \quad$ Desvio de freqüência utilizado na técnica LDV [Hz]

$G_{\varphi} \quad$ Fluxo de quantidade de movimento angular $\left[\mathrm{kg} \cdot \mathrm{m}^{2} / \mathrm{s}^{2}\right]$

$G_{x} \quad$ Fluxo de quantidade de movimento axial $\left[\mathrm{kg} \cdot \mathrm{m} / \mathrm{s}^{2}\right]$

$h(r) \quad$ Coeficiente local de troca de calor na placa $\left[\mathrm{W} / \mathrm{m}^{2} \mathrm{~K}\right]$

$H$ Distância jato-placa [mm]

l Corrente Elétrica [A]

$k \quad$ Condutividade térmica do $\operatorname{ar}[\mathrm{W} / \mathrm{m} . \mathrm{K}]$

$L \quad$ Comprimento da folha de aço inoxidável [mm]

Mo Fluxo de quantidade de movimento linear por unidade de Massa $\left[\mathrm{m}^{4} / \mathrm{s}^{2}\right.$ ]

N Número de ciclos no sinal em medição com LDV

$\mathrm{Nu}(r) \quad$ Número de Nusselt local

$N u_{\text {stag }}$ Número de Nusselt no ponto de estagnação

q" $\quad$ Fluxo de calor por unidade de área $\left[\mathrm{W} / \mathrm{m}^{2}\right]$

$Q \quad$ Vazão volumétrica $\left[\mathrm{m}^{3} / \mathrm{h}\right]$

$r \quad$ Coordenada radial

$R \quad$ Raio do tubo do jato [mm]

$\operatorname{Re} \quad$ Número de Reynolds

$S \quad$ Número de Swirl

$T(r) \quad$ Temperatura local na superfície da placa $[\mathrm{K}]$

$U \quad$ Velocidade Axial [m/s] 
$\bar{U} \quad$ Velocidade axial média $[\mathrm{m} / \mathrm{s}]$

$U_{j} \quad$ Velocidade Média de saída no tubo $[\mathrm{m} / \mathrm{s}]$

$u \quad$ Flutuação de velocidade axial [m/s]

$u^{\prime} \quad$ Velocidade axial r.m.s., $\sqrt{\bar{u}^{2}}[\mathrm{~m} / \mathrm{s}]$

$V \quad$ Velocidade Radial $[\mathrm{m} / \mathrm{s}]$

$\bar{V} \quad$ Velocidade radial média $[\mathrm{m} / \mathrm{s}]$

$v \quad$ Flutuação de velocidade radial [m/s]

$v^{\prime} \quad$ Velocidade radial r.m.s., $\sqrt{v^{2}}[\mathrm{~m} / \mathrm{s}]$

$W \quad$ Velocidade Tangencial $[\mathrm{m} / \mathrm{s}]$

$\bar{W} \quad$ Velocidade tangencial média $[\mathrm{m} / \mathrm{s}]$

w Flutuação de velocidade tangencial $[\mathrm{m} / \mathrm{s}]$

$w^{\prime} \quad$ Velocidade tangencial r.m.s., $\sqrt{w^{2}}[\mathrm{~m} / \mathrm{s}]$

$x \quad$ Coordenada vertical (axial) a partir da saída do jato

y Coordenada vertical (axial) a partir da superfície da placa

\section{Símbolos Gregos}

$\theta \quad$ Coordenada circunferencial

$\mu \quad$ Viscosidade dinâmica do ar [kg/m.s] 